\title{
Log10 Minimum Angle of Resolution
}

National Cancer Institute

\section{Source}

National Cancer Institute. Log10 Minimum Angle of Resolution. NCI Thesaurus. Code C142351.

The base-10 log arithm of the minimum angle of resolution achieved by an individual during testing. 